\title{
A Maximum a Posteriori Approach to Beamforming in the Presence of Calibration Errors
}

\author{
A. SWINDLEHuRst \\ Dept. of Elec. \& Comp. Engineering \\ Brigham Young University \\ Provo, UT 84602
}

\begin{abstract}
The performance of DF-based beamformers is seriously degraded in situations where the array is imprecisely calibrated, or when the spatial coherence of the signal wavefronts is perturbed. When the calibration errors or perturbation may be characterized by a set of parameters drawn from a known Gaussian distribution, a maximum a posteriori (MAP) estimator may be used to separately estimate the directions of arrival and the perturbation parameters, resulting in essentially an on-line auto-colibration. This paper examines the improvement that results from using the MAP auto-calibrated steering vectors in standard $D F$ based beamformers to estimate the received signal waveforms and suppress unwanted interference. For the special case of additive unstructured calibration errors and uncorrelated signals, it is shown that the MAP beamformer is similar in form to so-called "subspace corrected" approaches.
\end{abstract}

\section{Introduction}

All methods for direction-finding (DF) and DF-based beamforming rely on the availability of information about the array response, and assume the signal wavefronts have perfect spatial coherence. Depending on the degree to which the actual response or wavefronts differ from their nominal values, DF and beamformer performance may be significantly degraded. To account for these types of perturbations, a slightly generalized model for the array response will be considered in this paper. The response will be parameterized not only by the directions of arrival (DOAs) of the signals, but also by a vector of perturbation or "nuisance" parameters that describe deviations of the response from its nominal value. These parameters can include, for example, displacements of the antenna elements from their nominal positions, uncalibrated receiver gain and phase offsets, etc.. With such a model, a natural approach is to attempt to estimate the unknown nuisance parameters simultaneously with the signal parameters. Such methods are referred to as auto-calibration techniques, and have been proposed by a number of authors, including $[1,2,3,4]$ among many others.

When auto-calibration techniques are employed, it is critical to determine whether both the signal and nuisance parameters are identifiable. In certain cases they are not; for example, one cannot uniquely estimate both DOAs and sensor phase characteristics (unless of course additional information is available, such as sources in known locations, etc.). The identifiability problem can be alleviated if the perturbation parameters are assumed to be drawn from some known a priori distribution. While this itself represents a form of additional information, it has the advantage of allowing an optimal maximum a posteriori (MAP) solution to the problem to be formulated. In [4] it is shown that, by using an asymptotically equivalent approximation to the resulting MAP criterion, the estimation of the signal and nuisance parameters can be decoupled, leading to a significant simplification of the problem.

Presumably, any of the above auto-calibration methods would provide not only improved DOA estimates, but also calibration information that would be useful in beamformer implementation. In this paper, beamformer performance is investigated for the case where the optimal MAP perturbation parameter estimates of [4] are used to update the array calibration. Simulations demonstrate that such an approach can result in a significant performance improvement, measured using either interference rejection capability or mean-squared error. In addition, for simple additive unstructured calibration errors, the MAP approach is shown in certain cases to yield a beamformer similar to the subspace corrected algorithms described in $[5,6]$.

\section{Mathematical Model and Algorithms}

The response of an arbitrary array of $m$ sensors for a given DOA $\theta$ will be denoted by the $m$-vector $a(\theta, \rho)$, which is parameterized by a vector $\rho \in \mathbb{R}^{p}$ that describes the array perturbation. The array output is then modeled by the following familiar equation:

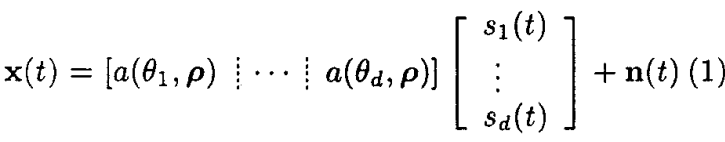

$$
\begin{aligned}
& =\mathbf{A}(\theta, \rho) \mathbf{s}(t)+\mathbf{n}(t),
\end{aligned}
$$

where $\mathbf{s}(t)$ and $\mathbf{n}(t)$ represent the received signals and noise, respectively. It will be assumed that for a given collect, $N$ samples are taken from the array. Both $\mathbf{s}(t)$ and $\mathbf{n}(t)$ are assumed to be temporally white zero-mean complex Gaussian random processes, with covariances given by $\sigma^{2} \mathbf{I}$ and $\mathbf{P}$, respectively. The perturbation term $\rho$ is also assumed to be drawn from a Gaussian distri- 
bution with known mean $\rho_{0}$ (corresponding to the nominal, unperturbed array response) and covariance $\boldsymbol{\Omega}$.

Given the above, the covariance of the array output and its eigendecomposition may be written as

$$
\mathbf{R}=\mathbf{A}(\boldsymbol{\theta}, \rho) \mathbf{P A}^{*}(\theta, \rho)+\sigma^{2} \mathbf{I}=\mathbf{E}_{s} \boldsymbol{\Lambda}_{s} \mathbf{E}_{s}^{*}+\sigma^{2} \mathbf{E}_{n} \mathbf{E}_{n}^{*}
$$

where $\boldsymbol{\Lambda}_{s}$ contains the $d$ largest eigenvalues, and the columns of the $m \times d$ matrix $\mathbf{E}_{s}$ are the corresponding unit-norm eigenvectors. Similarly, the columns of $\mathbf{E}_{n}$ are the $m-d$ eigenvectors corresponding to $\sigma^{2}$.

\subsection{An Asymptotic MAP Estimator}

In [4], it is shown that estimates of $\theta$ and $\rho$ asymptotically equivalent to those from the exact MAP estimator may be obtained by setting ${ }^{1}$

$$
\begin{aligned}
& \hat{\boldsymbol{\theta}}=\arg \min _{\theta} \mathbf{a}_{0}^{*} \hat{\mathbf{M}} \mathbf{a}_{0}-\hat{\mathbf{f}}^{T} \hat{\boldsymbol{\Gamma}}^{-1} \hat{\mathbf{f}} \\
& \hat{\boldsymbol{\rho}}=\rho_{0}-\boldsymbol{\Gamma}^{-1} \mathbf{f}
\end{aligned}
$$

where

$$
\begin{aligned}
\mathbf{a}_{0} & =\operatorname{vec}\left(\mathbf{A}\left(\theta, \rho_{0}\right)\right), \hat{\mathbf{M}}=\hat{\mathbf{U}}^{T} \otimes\left(\hat{\mathbf{E}}_{n} \hat{\mathbf{E}}_{n}^{*}\right) \\
\hat{\mathbf{U}} & =\hat{\sigma}^{-2} \hat{\mathbf{A}}^{\dagger} \hat{\mathbf{E}}_{s} \tilde{\mathbf{\Lambda}}^{2} \hat{\mathbf{\Lambda}}_{s}^{-1} \hat{\mathbf{E}}_{s}^{*} \hat{\mathbf{A}}^{\dagger *}, \hat{\mathbf{f}}=\operatorname{Re}\left\{\hat{\mathbf{D}}_{\rho}^{*} \hat{\mathbf{M}} \mathbf{a}_{0}\right\}(6) \\
\hat{\mathbf{\Gamma}} & =\operatorname{Re}\left\{\hat{\mathbf{D}}_{\rho}^{*} \hat{\mathbf{M}} \hat{\mathbf{D}}_{\rho}+\frac{1}{2 N} \boldsymbol{\Omega}^{-1}\right\} \\
\mathbf{D}_{\rho} & =\left.\left[\frac{\partial \mathbf{a}(\boldsymbol{\theta}, \boldsymbol{\rho})}{\partial \rho_{1}}, \ldots, \frac{\partial \mathbf{a}(\boldsymbol{\theta}, \boldsymbol{\rho})}{\partial \rho_{p}}\right]\right|_{\theta, \rho_{0}}
\end{aligned}
$$

and where $\hat{\sigma}^{2}$ and $\hat{\mathbf{A}}$ are "consistent" estimates determined from some initial estimation step. The above approach is quite general in that, by proper choice of $\rho$, it can be applied to arbitrary types of model errors. Another key advantage is that estimation of $\theta$ and $\rho$ is decoupled; a search is required only for the $d$ DOA parameters in $\theta$, and not for $\rho$ (which is calculated directly given $\hat{\boldsymbol{\theta}}$ ). Other properties of the algorithm are outlined in [4].

\subsection{Optimal Beamformers}

The minimum mean squared error (MSE) beamformer weights are easily shown to be

$$
\mathbf{W}_{M S E}=\mathbf{R}^{-1} \mathbf{R}_{x s}=\mathbf{R}^{-1} \mathbf{A}(\theta, \rho) \mathbf{P}
$$

When the desired signal is uncorrelated with the interference, $\mathbf{P}$ is diagonal and the minimum MSE solution is

\footnotetext{
${ }^{1}$ Strictly speaking, the equivalence of the above estimator and the optimal MAP approach only holds for first order errors $\rho-\rho_{0}$ that are "of the same order" as the finite sample effects of the noise. In other cases (particularly those model errors are dominant), a different approach should be used. For more details, see $[4,7]$.
}

just a scaled version of the so-called minimum variance distortionless response (MVDR) beamformer:

$$
\mathbf{W}=\frac{\mathbf{R}^{-1} a(\hat{\theta})}{a^{*}(\hat{\theta}) \mathbf{R}^{-1} a(\hat{\theta})}
$$

In the general case where the signal and interference are correlated, the optimal weights depend on the signals themselves through $\mathbf{R}_{x s}$ or $\mathbf{P}$, and thus they cannot be used directly (i.e., without a training sequence, for example). In the approach of [8], the quantities $\mathbf{P}$ and $\mathbf{R}$ in (9) are replaced by their structured $\mathrm{ML}$ estimates:

$$
\hat{\mathbf{P}}_{s}=\mathbf{A}_{0}^{\dagger}\left(\hat{\mathbf{R}}-\hat{\sigma}^{2} \mathbf{I}\right) \mathbf{A}_{0}^{\dagger *}, \hat{\mathbf{R}}_{s}=\mathbf{A}_{0} \hat{\mathbf{P}}_{s} \mathbf{A}_{0}^{*}+\hat{\sigma}^{2} \mathbf{I}
$$

where $\mathbf{A}_{0}=\mathbf{A}\left(\hat{\boldsymbol{\theta}}, \rho_{0}\right),(\cdot)^{\dagger}$ denotes a (left) pseudoinverse, and $\hat{\mathbf{R}}$ is a sample estimate of $\mathbf{R}$.

Since calibration errors were not addressed in [8], the nominal model $\rho_{0}$ was used to calculate the beamformer weights. Nevertheless, the method performs well when calibration errors are present, as recently demonstrated in [9]. On the other hand, the MVDR approach is well known to be hyper-sensitive to array perturbations, especially a.t high SNR. While ad hoc methods employing artificial noise injection have been used to combat this problem, other techniques based on subspace corrected (SC) weights have found success in experimental systems $[5,6]$. In these approaches, the $\mathbf{R}^{-1}$ term in (10) is replaced by $\mathbf{E}_{s} \boldsymbol{\Lambda}_{s}^{-1} \mathbf{E}_{s}^{*}$. This is equivalent to projecting $a(\hat{\theta})$ onto the signal subspace prior to forming the MVDR weights.

One of the goals of this paper is to study the improvement that results from using the method of [8] with $\mathbf{A}(\hat{\boldsymbol{\theta}}, \hat{\boldsymbol{\rho}})$ rather than $\mathbf{A}\left(\hat{\boldsymbol{\theta}}, \rho_{0}\right)$, where $\hat{\boldsymbol{\rho}}$ is obtained from the MAP estimator in (4). This approach will be referred to as the MAP beamformer in the sequel. In the next section, an interesting connection is made between the MAP beamformer and the SC-MVDR method. In particular, it is shown that for simple unstructured array error:s and uncorrelated signals, the SC-MVDR and MAP weights have a very similar form.

\section{Some Special Cases}

For the moment, consider the following simple unstructured model for the perturbed array response:

$$
\begin{aligned}
\mathbf{A}(\boldsymbol{\theta}, \boldsymbol{\rho}) & =\mathbf{A}(\boldsymbol{\theta})+\tilde{\mathbf{A}} \\
\boldsymbol{\rho} & =\left[\begin{array}{l}
\operatorname{Re}\{\operatorname{vec}(\tilde{\mathbf{A}})\} \\
\operatorname{Im}\{\operatorname{vec}(\tilde{\mathbf{A}})\}
\end{array}\right],
\end{aligned}
$$

where the columns of $\tilde{\mathbf{A}}$, denoted $\tilde{a}_{i}$, are modeled as zero mean Gaussian random vectors with moments

$$
\mathbf{E}\left[\tilde{a}_{i} \tilde{a}_{i_{k}}^{*}\right]=\nu_{i k} \mathbf{I}, \quad \mathbf{E}\left[\tilde{a}_{i} \tilde{a}_{k}^{T}\right]=0 \quad i, k=1, \cdots, d .
$$


This model corresponds to an additive, circularly symmetric complex array perturbation that is uncorrelated from sensor to sensor, but possibly $\theta$-dependent. It is easy to verify that under these assumptions, the covariance of $\rho$ is given by

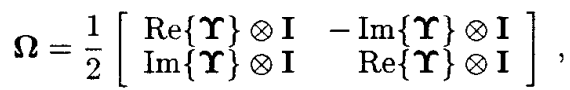

where the $i, k^{t h}$ element of the matrix $\Upsilon$ is $\nu_{i k}$.

It is interesting to examine the form of the MAP estimate $\hat{\rho}$ for this case. To begin with, note that for the above model $\rho_{0}=0$ and $\hat{\mathbf{D}}_{\rho}=\left[\begin{array}{ll}\mathbf{I} & j \mathrm{I}\end{array}\right]$, where $\mathbf{I}$ is $m d \times m d$. Thus, $\hat{\rho}=-\Gamma^{-1} \mathbf{f}$, and

$$
\begin{aligned}
& \boldsymbol{\Gamma}=\left[\begin{array}{lr}
\operatorname{Re}\left\{\hat{\mathbf{M}}+\frac{1}{N} \mathbf{\Upsilon}^{-1} \otimes \mathbf{I}\right\} & -\operatorname{Im}\left\{\hat{\mathbf{M}}+\frac{1}{N} \mathbf{\Upsilon}^{-1} \otimes \mathbf{I}\right\} \\
\operatorname{Im}\left\{\hat{\mathbf{M}}+\frac{1}{N} \mathbf{\Upsilon}^{-1} \otimes \mathbf{I}\right\} & \operatorname{Re}\left\{\hat{\mathbf{M}}+\frac{1}{N} \mathbf{\Upsilon}^{-1} \otimes \mathbf{I}\right\}
\end{array}\right] \\
& \mathbf{f}=\left[\begin{array}{l}
\operatorname{Re}\left\{\hat{\mathbf{M}} \mathbf{a}_{0}\right\} \\
\operatorname{Im}\left\{\hat{\mathbf{M}} \mathbf{a}_{0}\right\}
\end{array}\right] .
\end{aligned}
$$

Using the fact that, for any invertible matrix $\mathbf{Z}$,

$$
\left[\begin{array}{lr}
\operatorname{Re}\{\mathbf{Z}\} & -\operatorname{Im}\{\mathbf{Z}\} \\
\operatorname{Im}\{\mathbf{Z}\} & \operatorname{Re}\{\mathbf{Z}\}
\end{array}\right]^{-1}=\left[\begin{array}{lr}
\operatorname{Re}\left\{\mathbf{Z}^{-1}\right\} & -\operatorname{Im}\left\{\mathbf{Z}^{-1}\right\} \\
\operatorname{Im}\left\{\mathbf{Z}^{-1}\right\} & \operatorname{Re}\left\{\mathbf{Z}^{-1}\right\}
\end{array}\right]
$$

it is easy to show that

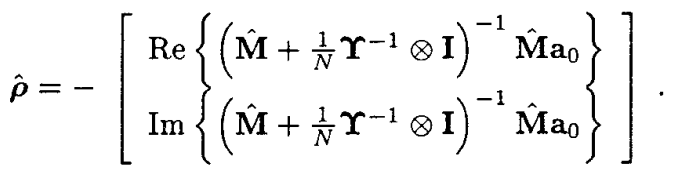

A further simplification of (16) is possible that is quite revealing. Using the definition of $\hat{\mathbf{M}}$ in (5), note that

$$
\begin{aligned}
& \left(\hat{\mathbf{M}}+\frac{1}{N} \mathbf{\Upsilon}^{-1} \otimes \mathbf{I}\right)^{-1} \\
& =\left(\hat{\mathbf{U}}^{T}+\frac{1}{N} \boldsymbol{\Upsilon}^{-1}\right)^{-1} \otimes\left(\hat{\mathbf{E}}_{n} \hat{\mathbf{E}}_{n}^{*}\right)+N \mathbf{\Upsilon} \otimes\left(\hat{\mathbf{E}}_{s} \hat{\mathbf{E}}_{s}^{*}\right)
\end{aligned}
$$

Multiplying this equation on the right by $\hat{\mathbf{M}} \mathbf{a}_{0}$ and simplifying then yields

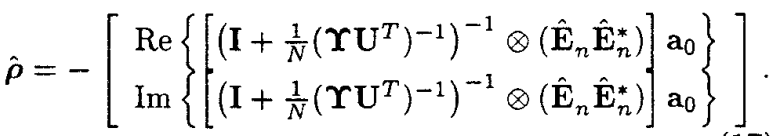

Finally, using (12) and properties of the Kronecker product, the MAP estimate of the array response becomes

$$
\mathbf{A}(\hat{\boldsymbol{\theta}}, \hat{\boldsymbol{\rho}})=\mathbf{A}(\hat{\boldsymbol{\theta}})-\hat{\mathbf{E}}_{n} \hat{\mathbf{E}}_{n}^{*} \mathbf{A}(\hat{\boldsymbol{\theta}})\left(\mathbf{I}+\frac{1}{N}\left(\mathbf{\Upsilon} \mathbf{U}^{T}\right)^{-1}\right)^{-1} .
$$

The key point of interest is that, if $\mathbf{\Upsilon}^{-1} / N \rightarrow 0$, then the MAP estimate of the array response converges to a subspace corrected version of the nominal response:

$$
\lim _{\Upsilon \rightarrow 1 / N \rightarrow 0} \mathbf{A}(\hat{\boldsymbol{\theta}}, \hat{\boldsymbol{\rho}})=\hat{\mathbf{E}}_{s} \hat{\mathbf{E}}_{s}^{*} \mathbf{A}(\hat{\boldsymbol{\theta}}) \text {. }
$$

Furthermore, if the estimated MAP array response is used in (10), the MVDR beamformer (10) will converge to the SC-MVDR approach. The condition $\mathbf{\Upsilon}^{-1} / N \rightarrow 0$ occurs either with a large data sample, or when the array perturbation is large. In either case, the information provided by the prior distribution of $\rho$ is of little value, and is essentially ignored by the MAP criterion. This observation provides some theoretical justification for the SC-MVDR technique, which previously had been derived using ad hoc (but well motivated) reasoning. However, in cases where the prior cannot be neglected, using SC response vectors for beamforming will not be optimal and significant degradation can result. This is seen in the simulation examples described later.

\subsection{Gain and Phase Errors}

For arrays composed of nominally identical elements, a common approach used to describe deviations in the array response attempts to model the non-uniform gain and phase effects of the receiver electronics behind each antenna element. In this model, the nominal response is perturbed by an unknown complex diagonal matrix:

$$
\mathbf{A}(\theta, \rho)=\mathbf{G A}(\boldsymbol{\theta}), \quad \rho=\left[\begin{array}{l}
\operatorname{Re}\{\mathbf{g}\} \\
\operatorname{Im}\{\mathbf{g}\}
\end{array}\right]
$$

where $\mathbf{g}=\operatorname{diag}\{\mathbf{G}\}$. The mean of the distribution for $\rho$ in this case is given by $\rho_{0}=\left[\begin{array}{ll}\mathbf{e}^{T} & 0\end{array}\right]^{T}$, where $\mathbf{e}$ is an $m \times 1$ vector of ones. For simplicity, in this discussion the covariance of $\rho$ will be assumed to be $\Omega=\left(\sigma_{a}^{2} / 2\right) \mathbf{I}$, which implies that the individual gain and phase errors are all mutually independent and identically distributed.

The derivation of the MAP estimate of $\rho$ and hence $g$ is straightforward but somewhat cumbersome, and thus will not be presented here. However, the result is quite simple, and is given by

$$
\begin{aligned}
& \hat{\mathbf{g}}=\left(\mathbf{I}+\sigma_{a}^{2} N \mathbf{Z}\right)^{-1} \mathbf{e} \\
& \mathbf{Z}=\left[\sum_{i, k=1}^{d} u_{k i} \bar{a}\left(\hat{\theta}_{i}\right) a^{T}\left(\hat{\theta}_{k}\right)\right] \odot\left(\hat{\mathbf{E}}_{n} \hat{\mathbf{E}}_{n}^{*}\right),
\end{aligned}
$$

where $u_{k i}$ is the $k, i^{\text {th }}$ element of $\hat{\mathbf{U}}, \overline{(\cdot)}$ denotes conjugation, and $\odot$ an element-wise (Hadamard) product. Note that for very small gain/phase errors where $\sigma_{a} \rightarrow 0$, $\hat{\mathbf{g}} \rightarrow \mathbf{e}$ and hence $\hat{\mathbf{G}} \rightarrow \mathbf{I}$ as expected.

\section{Simulation Results}

In this section, the performance of the MAP beamformer is studied by means of a number of simulation examples. The first example involves a nominally unitgain uniform linear array perturbed by an unstructured calibration error in the form of equation (11)-(14) with $\Upsilon=\sigma_{a}^{2} \mathbf{I}$ and $\sigma_{a}=0.2$. The array receives 100 samples of two $20 \mathrm{~dB}$ SNR uncorrelated Gaussian signals with 


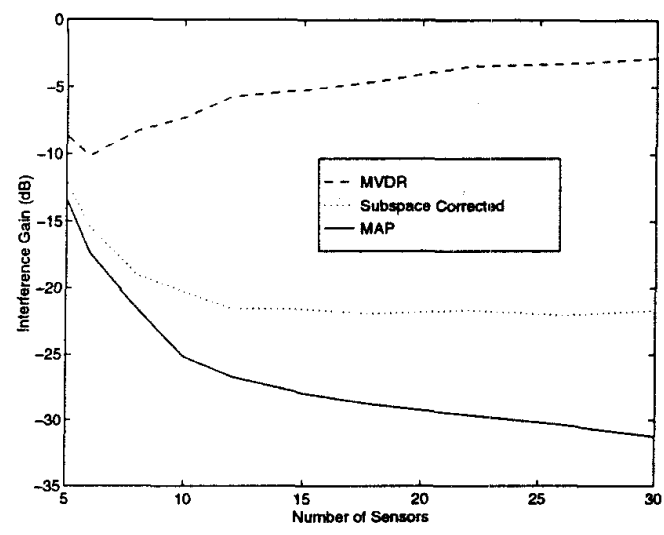

Figure 1: A Comparison of Beamformer Performance, Unstructured Calibration Errors

arrival angles of $5^{\circ}$ and $15^{\circ}$. Using DOA estimates from the optimal MAP estimator, the relative interference rejection capability of the MVDR, SC-MVDR, and MAP beamformers was calculated for various array sizes. The results are plotted in Figure 1 based on 500 independent trials. The plot shows the gain of the beamformer weights for the $5^{\circ}$ source in the direction of the $15^{\circ}$ interferer (normalized for a unit gain response at $5^{\circ}$ ). The subspace correction eliminates the signal cancelation effect of the MVDR approach, but the MAP beamformer provides a significant advantage, especially for larger arrays. The above simulation was repeated assuming receiver gain/phase errors as described by (19), also with $\sigma_{a}=0.2$, and a plot almost identical to Figure 1 was obtained. Algorithm performance is seen in this case to depend very little on the type of calibration error encountered.

When the signals arriving at the array are highly correlated, interference rejection is no longer an appropriate performance criterion. In such cases, an optimal beamformer will attempt to combine correlated arrivals with the desired signal to improve the quality of the resulting estimate, as measured using (for example) meansquared error. To examine beamformer performance for the case of correlated signals, a two-ray multipath channel was simulated for various relative delays. A miscalibrated 5-element linear array was assumed to receive a random QPSK signal from $-6^{\circ}$, as well as a slightly delayed copy of the signal from $6^{\circ}$. Both arrivals had an SNR of $0 \mathrm{~dB}$, and the array was again perturbed according to (11)-(14) with $\Upsilon=\sigma_{a}^{2} I$ and $\sigma_{a}=0.15$. For each trial, MAP DOA estimates were obtained based on 75 samples from the array, and normalized RMS signal errors were computed. The results are plotted in Figure 2 for various relative delays between the two arrivals. The "uncompensated" approach corresponds to the method of [8] implemented with $\mathbf{A}\left(\hat{\boldsymbol{\theta}}, \boldsymbol{\rho}_{0}\right)$ rather than $\mathbf{A}(\hat{\boldsymbol{\theta}}, \hat{\boldsymbol{\rho}})$ as in the MAP beamformer. The minimum MSE curve was obtained using a known 75-sample training sequence to compute the optimal weights, and was included to give

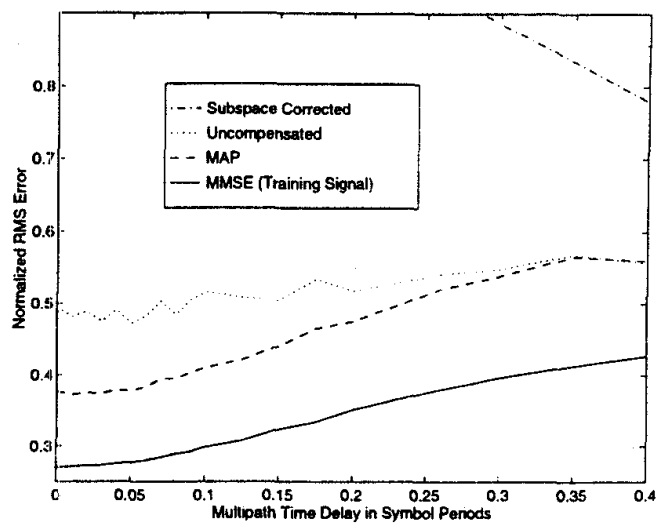

Figure 2: Floot MSE Performance of Various Beamformers for a Multipath Channel

an idea of the "best possible" performance.

While the SC-MVDR approach can to some degree compensate for array perturbations, it cannot eliminate signal cancelation due to the presence of a correlated arrival, and its performance in this case is quite poor. For small delays, correcting for calibration errors yields a 25-30\% improvement in RMS error, which translates into a reduction in symbol error rate of approximately a factor of 6 (from .041 to .007) for this example.

\section{References}

[1] A. Paulraj and T. Kailath, "Direction-of-Arrival Estimation by Eigenstructure Methods with Unknown Sensor Gain and Phase", In Proc. ICASSP, pages 17.7.1-17.7.4, 1985.

[2] Y. Rockah and P. M. Schultheiss, "Array" Shape Calibration Using Sources in Unknown Locations - Part I: Far-Field Sources", IEEE Trans. on ASSP, 35:286-299, March 1987.

[3] A. J. Weiss and B. Friedlander, "Array Shape Calibration Using Sources in Unknown Locations - A Maximum Likelihood Approach", IEEE Trans. on ASSP, 37:1958-1966, Dec. 1989.

[4] M. Viberg and A. Swindlehurst. "A Bayesian Approach to Auto-Calibration for Parametric Array Signal Processing", IEEE Trans. on Sig. Proc., 42:3495-3507, Dec. 1994.

[5] R. Schmidt and R. Franks, "Multiple Source DF Signal Processing: An Experimental System", IEEE Trans. on Ant. and Prop., 34:281-290, March 1986.

[6] B. Wahlberg, I. Mareels, and I. Webster, "Experimental and Theoretical Comparison of some Algorithms for Beamforming in Single Receiver Adaptive Arrays". IEEE Trans. on Ant. and Prop., 39:21-28, Jan. 1991.

(7) M. Viberg and A. Swindlehurst, "Analysis of the Combined Effects of Finite Samples and Model Errors on Array Processing Performance", IEEE Trans. on Sig. Proc., 42:3073-3083, Nov. 1994.

[8] B. Ottersten, R. Roy, and T. Kailath, "Signal Waveform Estimation in Sensor Array Processing", In Proc. $23^{\text {rd }}$ A silomar Conference, pages 787-791, Nov. 1989.

[9] J. Yang and A. Swindlehurst. "The Effects of Array Calibration Errors on DF-Based Signal Copy Performance", IEEE Trans. on Sig. Proc., 43:2724-2732, Nov. 1995. 